\section{BACTERIAL SECRETION}

\section{Autotransporters monomers and multimers}
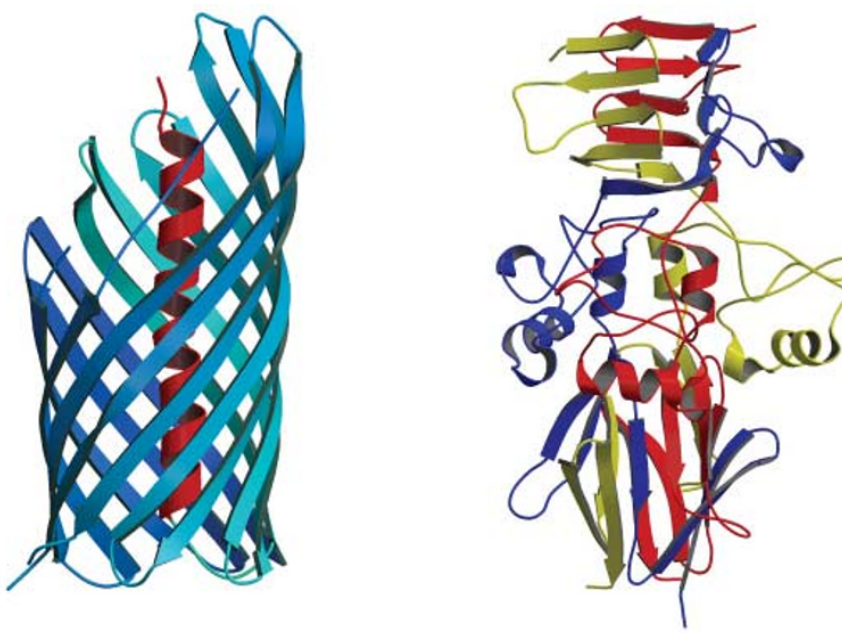

Ribbon diagrams of the translocator domain of the classic autotransporter protein NalP (left) and the primary binding domain of $\mathrm{Hia}$ (right), a member of the recently recognized trimeric autotransporter subfamily. The helix inserted into the pore of NaIP (left) is shown in red. Each subunit of the Hia adhesin (right) is colour-coded differently. Image courtesy of $\mathrm{C}$. Oomen, Birkbeck College, London, UK.
Protein secretion from Gram-negative bacteria involves transport across both the inner and outer membranes. Now, two high-resolution structures in EMBO Journal shed light on the mechanisms of secretion by the autotransporter system and the role of these secreted proteins in virulence.

Autotransporters consist of three domains: an $\mathrm{N}$-terminal signal peptide, which directs export of the precursor protein across the inner membrane before being cleaved; a C-terminal translocator, or $\beta$, domain, which inserts into the outer membrane; and a passenger domain, which is surface localized and often released from the bacterium.

Piet Gros and colleagues have solved a high-resolution X-ray crystal structure of the translocator domain of NalP from Neisseria meningitidis. The structure reveals a 12-stranded $\beta$-barrel with a hydrophilic pore of $10 \times 12.5 \AA$ - a classical structure for outer-membrane proteins. But, unusually, the pore is filled with an N-terminal $\alpha$-helix. Previous studies have proposed two distinct models for the protein-translocation process: transportation of the passenger domain through a pore formed by a single translocator domain; and transportation of the passenger domain through a pore formed by a multimer of translocator domains. The presence of hydrophobic residues on the exterior of the $\beta$-barrel seems inconsistent with the multimeric model; however, on the basis of the crystal structure the monomeric model would involve translocation of an unfolded protein. For these reasons, they propose an alternative mechanism that requires the Omp85 complex (which is required for the assembly of integral outer-membrane proteins).

In a separate study, Gabriel Waksman, Joseph St Geme and colleagues report the X-ray crystal structure of the HiaBD1 region of the passenger domain of the Haemophilus influenzae Hia protein — an adhesin that is primarily responsible for the binding of $H$. influenzae to host cell receptors. Unlike NalP, the passenger

\title{
A spot of light training?
}

Clock genes, which are central to circadian regulation, have been found in all organisms. How clocks are synchronized with, or entrained to, a 24-h day, and adjusted to different seasonal daylengths is, however, still a puzzle. A new report published in Current Biology reveals that entrainment of the model fungus Neurospora crassa clock is more complex than previously thought. Rather than a simple transcriptional feedback regulatory loop, translation and protein stability also impinge on clock control.

Light, the most important environmental training signal, can switch clock genes on, and promote degradation of clock proteins. Multiple input proteins feed light signalling into the N. crassa clock, but entrainment is crucially dependent on the frq gene. frq is negatively regulated by FRQ protein abundance, but because frq is strongly induced by a pulse of light, it was previously thought that the fungal clock was lightdriven, and that the effects of light could over-ride autorepression by the FRQ protein.
Here, Tan et al. show that during a 24-h day the frq gene is strongly induced at 'lights-on', and decreases after 'lights-off', so the clock does seem to be light-driven. What happens to the FRQ protein though, during daily cycles?

Using 24-h days with different photoperiods - short days/long nights or short nights/long days - FRQ abundance was upregulated after lights-on, but the timing of this was found to depend on the length of the night. Long nights resulted in a rapid upsurge in abundance of the FRQ protein after lights-on, whereas after short nights, abundance of the FRQ protein was upregulated after lights-on, but with a considerable delay. This indicates that transcription and translation at the frq locus have become dissociated by the length of the photoperiod. Plus, levels of the FRQ protein declined after lights-off at different rates, dependent on the photoperiod. Halfmaximal levels were always reached at around midnight, whatever the photoperiod, within a 24-h day.
To attempt to reconcile this with the known response of spore formation (conidiation), which always occurs $7 \mathrm{~h}$ after lights-off in $50 \%$ light/dark cycles of varying lengths, conidiation was carried out under the same conditions. Using a 24-h day with varying day/night lengths, conidiation always occurred around midnight — when the FRQ protein was at half-maximal levels independent of the photoperiod. It seems that FRQ abundance regulates conidiation.

Expression of the clock component protein FRQ integrates environmental history depending on the photoperiod, the FRQ protein declines at different rates - whereas the frq gene is always upregulated in response to light. So the N. crassa clock is not, after all, solely driven by light, but fluctuates during a light/dark cycle like the clocks of higher organisms. Biochemistry and genetics will now be needed to tease out how FRQ degradation is modulated.

(10) References and links

Susan Jones ORIGINAL RESEARCH PAPER Tan, Y. et al. Entrainment dissociates transcription and translation of a circadian clock gene in Neurospora. Curr. Biol. 14, 433-438 (2004) FURTHER READING Liu, Y. Molecular mechanisms of entrainment in the Neurospora circadian clock. J. Biol. Rhythms 18, 195-205 (2003) WEB SITES

Till Roenneberg's laboratory: http://www.imp-muenchen.de/?chronobiology Encyclopedia of Life Sciences: http://www.els.net Circadian rhythms in Neurospora 
